\title{
Cumulative Harm, Products Liability and Bilateral Care
}

Andrew F. Daughety and Jennifer F. Reinganum 


\title{
Cumulative Harm, Products Liability, and Bilateral Care
}

\author{
Andrew F. Daughety, Vanderbilt University and Jennifer F. Reinganum, \\ Vanderbilt University
}

Send correspondence to: Jennifer F. Reinganum, Department of Economics and Law School, Vanderbilt University, VU Station B \#351819, 2301 Vanderbilt Place, Nashville, TN 37235-1819; E-mail: jennifer.f.reinganum@vanderbilt.edu

We extend consideration of cumulative harm in products liability to the case of bilateral care. For this specification, the level of care and the level of output chosen by the firm are inextricably interrelated, and different liability regimes yield different combinations of care and output. As in the case of bilateral care with proportional harm, strict liability by itself leads to moral hazard on the part of the consumer, but strict liability with a defense of contributory negligence on the part of the consumer is now a resilient (that is, both robust and convergent) liability rule when harm is cumulative. (JEL: K13, L15, D42)

\section{Introduction}

In the traditional law and economics model of products liability with observable care, expected harm is proportional to the consumer's use of the product (see e.g., Shavell, 1980, 2007; Landes and Posner, 1985). As a consequence, the socially efficient levels of care for both the firm and the

This paper was primarily written while both authors were Visiting Scholars at Stanford Law School (we especially thank Mitch Polinsky for providing support for our visit) and then revised while both authors were visitors at the Institute for Advanced Study in Toulouse (we especially thank Paul Seabright for providing support for our visit). This paper and a companion paper, Daughety and Reinganum (2014), formed the basis of Reinganum's ALEA Presidential Address in May, 2012, at Stanford Law School. We thank the Editor, Abraham Wickelgren, and an anonymous referee for constructive suggestions. 
consumer (if care is bilateral) are independent of the level of output in the market. When only the firm can affect the expected harm through its choice of care (care is unilateral), then socially efficient care levels can be achieved in equilibrium by employing various liability rules, including strict liability, no liability, and negligence. When care is bilateral, then strict liability must be augmented with a defense of contributory negligence to restore incentives for socially efficient care; the regimes of no liability and negligence continue to generate socially efficient care without modification.

The fact that socially efficient and equilibrium care levels are independent of the level of consumption means that the tort system can focus on optimizing "product performance" (i.e., the level of care and resulting product safety) while other agencies or bodies of law can focus on "market performance" (i.e., the level of output and resulting total surplus). We do in fact observe divided responsibilities in our complex system of regulatory and common law. The traditional products liability model suggests that divided responsibilities do not entail a social loss, since no coordination among policies is required to achieve the joint socially efficient combination of care and output.

In a previous paper (Daughety and Reinganum, 2014, hereafter DR2014), we show that when the consumer's expected harm is cumulative in consumption (i.e., expected harm increases more-than-proportionally in the amount consumed $)^{1}$ and care is unilateral, then the socially efficient levels of care and output are "entangled." That is, there is a different efficient level of care for each given level of output, and a different efficient level of output for each given level of care. Profit-maximizing levels of output and care are similarly entangled. We show that strict liability and no liability

1. Marino (1988) introduces the notion of cumulative harm (and its opposite, tolerance). See DR2014 for an extensive discussion of goods wherein expected harm is likely to be cumulative. These include pharmaceuticals (e.g., the Exelon patch and opiod pain relievers) and over-the-counter medications (e.g., acetaminophen and NSAIDS) wherein the likelihood and severity of side effects increase with dosage; foods that may harbor bacteria in sufficient quantities to cause illness (e.g., chicken contaminated with Salmonella, ground beef contaminated with E. coli, and cold cuts or melon contaminated with listeria); goods involving exposure to chemicals or radiation that bioaccumulates in the body (e.g., products containing asbestos or lead, fish containing mercury, and CT scans or X-rays); goods with rapidly moving parts (such as motors) that deteriorate with wear; and activities that can be "tracked" (e.g., as in online environments), which can generate expected harm through loss of privacy. 
now generate different choices of care and output, while negligence simply devolves into strict liability. Although strict liability does not generate the overall socially efficient combination of care and output, it does generate socially efficient care for any given level of output (while no liability generates excessive care for any given level of output).

In DR2014 we define a resilient liability rule as one that displays two properties: (1) it is robust in the sense that it continues to work well even if the firm's choice of care is unobservable, and it is not unstable (in the way that negligence is, even with observable care); and (2) it is convergent in the sense that it responds appropriately to welfare-enhancing improvements in output undertaken by another agency or body of law tasked with optimizing market performance. In particular, as the level of output converges to its socially efficient level, a rule of strict liability causes the firm's choice of care to converge to its socially efficient level while a rule of no liability causes the firm's choice of care to diverge from its socially efficient level.

In this paper, we extend our model of products liability with cumulative expected harm to the case of bilateral care. We pose the model as one of sequential care-taking, wherein the firm takes care in manufacturing the product and the consumer subsequently takes care in using the product. We show that strict liability with a defense of contributory negligence on the part of the consumer results in the socially efficient levels of care for both the firm and the consumer for any given level of output (whereas no liability does not achieve this conditional efficiency). In particular, we find that, for any given level of output, the firm prefers to choose its care level so as to induce the consumer to subsequently meet her due-care requirement; this implies that the firm prefers to face strict liability rather than no liability. Consequently, strict liability with a defense of contributory negligence displays the qualities of robustness and convergence described above; it is the resilient liability rule.

Plan of the Paper: In Section 2, we provide a detailed review of related literature. In Section 3, we briefly review the proportional-harm model results, allowing for sequential choice of care. We then analyze the cumulative-harm model under bilateral sequential care-taking, and show that strict liability with a defense of contributory negligence is a resilient liability rule, whereas a rule of no liability is not resilient. In Section 4, we provide a brief summary and conclusion; the Appendix provides some of the more technical arguments. 


\section{Literature Review}

In this paper, we will employ a model of harm in which both the firm and the (representative) consumer can exert care to lower the expected harm (that is, we will use a "bilateral-care" model) and wherein the expected harm is cumulative in the sense that it is increasing at a more-than-proportional rate in the amount of the good consumed. Thus, the model we use has antecedents in the proportional-harm model (wherein expected harm is proportional to the amount of the good consumed) under both unilateral and bilateral care. It is also related to more recent work on models of cumulative harm with unilateral care. We will discuss these strands of the literature in turn.

As indicated in Section 1, when: (1) only the firm can exert care to reduce the expected harm (a unilateral-care model); (2) care is observable prior to purchase; (3) the cost of production-plus-care per unit of output is constant; and (4) the expected harm per unit of output consumed is constant (a proportional-harm model), then all standard liability regimes (strict liability, negligence, and no liability) yield the socially efficient level of care (Shavell, 1980; Landes and Posner, 1985). The firm's level of output need not be the socially efficient level if the firm has some market power, but since the socially efficient level of care is independent of the level of output in the proportional-harm model, the court can choose the liability regime without concern for the structure of the market (e.g., the degree of competitiveness).

When care is unilateral, but expected harm is cumulative, then the socially efficient levels of care and output are "entangled" (this is also true for the profit-maximizing levels of care and output). ${ }^{2}$ That is, there is a different efficient level of care for any given level of output, and a different efficient level of output for any given level of care. Moreover, the firm will face different harm-related costs under different liability rules. In particular, when the firm is subject to strict liability, then its choice of care will be

2. This interdependence is explored in early papers by Spence (1975) and Sheshinski (1976) wherein a monopolist chooses product quality rather than investment in care (and abstracting from issues of warranty or liability). More recent papers in law and economics wherein choices of output and care are interdependent include Polinsky (1980); Polinsky and Rogerson (1983), and Daughety and Reingnaum (2006); this interdependence arises in these models for various reasons that are unrelated to cumulative harm. 
socially efficient for any given level of output since it will face the average expected harm per unit sold. However, it will produce too little output for any given level of care (due to its monopoly power). Therefore, when harm is cumulative the firm will ultimately produce too little output and exert too little care under strict liability (Marino, 1988; Spulber, 1989; DR2014). ${ }^{3}$ On the other hand, when the firm is subject to no liability, then the consumer's demand will reflect this via her deduction of the marginal expected harm from her marginal willingness-to-pay for the good. Since the marginal expected harm exceeds the average expected harm when expected harm is cumulative, the firm will face higher liability-related harms when these are conveyed through the market behavior of the consumer than when they are conveyed directly through strict liability. In this case, the firm exerts excessive care for any given level of output, but produces even less output for any given level of care than does the firm facing strict liability. Under no liability, a firm with market power ultimately produces too little output, and that output is excessively safe (DR2014).

In addition, in DR2014 we find that the negligence regime is unstable in the sense that the firm, which prefers strict liability to no liability (due to the aforementioned reduction in the consumer's marginal willingness-to-pay at the rate of marginal expected harm), intentionally violates the negligence standard in order to provoke strict liability. For the quadratic expected harm function used therein we find that welfare is higher at the equilibrium under strict liability than at the equilibrium under no liability. All of these results point towards strict liability as the best liability regime, although it does not achieve the overall social-welfare optimum when firms have market power.

3. Marino (1988) considers a unilateral-care monopoly model under strict liability and no liability, and compares the associated market outcomes to the socially efficient outcome, obtaining the aforementioned interdependence between output and care; see also Spulber (1989). In DR2014, we focus not only on cumulative harm and unilateral care, but we also include negligence as a liability rule and obtain more comprehensive welfare rankings. In addition, we provide two versions of an oligopoly model wherein firms chose output levels: (1) the independent-harms model, wherein each firm's output is identifiable and the expected harm associated with one product does not depend on other firms' output levels and (2) the joint-harm model wherein the firms' outputs are indistinguishable and the expected harm is cumulative in the consumer's aggregate consumption of all products in the relevant market. In the independent-harms model, strict liability continues to be the resilient policy; in the joint-harm model a modified version of market-share liability (which is also a version of strict liability) is the resilient policy. 
In DR2014, we observe that, although there is inherent interdependency in the choices of care and output, the responsibilities for "product performance" (which encompasses the level of care taken and safety achieved) and "market performance" (which encompasses the level of prices and output) are not centralized, but rather are distributed across different government regulatory agencies and bodies of law. Rather than expecting any one court or agency to solve the entire problem, we argue that strict liability is a resilient policy in that it is stable (unlike negligence); it is robust to the possibility that consumers cannot observe the firm's care prior to purchase (unlike no liability); and as another agency tasked with optimizing market performance moves the output level toward the socially efficient level (e.g., through output subsidies), the firm's profit-maximizing level of care will converge along with output to the socially efficient level (again, unlike no liability, which results in increasingly excessive care). Thus, by adopting strict liability, the tort system can focus on product performance, anticipating that market performance will be optimized by another regulatory agency or body of law and legal precedent.

While strict liability is not problematic in a context wherein consumer care plays no role, it is highly problematic when consumer care is also important in avoiding harm. In particular, when the consumer is fully compensated for harm due to the use of the product, it is optimal for her to exert no effort on care. ${ }^{4}$ This has led, in the proportional-harm bilateralcare model, to considering the use of strict liability with a defense of contributory negligence. That is, the firm is strictly liable unless the consumer was contributorily negligent by taking less care than is specified by a duecare standard (e.g., Shavell, 1980; Landes and Posner, 1985, where it is implicitly assumed that both parties choose their levels of care simultaneously). We extend this result to the case of cumulative harm below, where we also modify the timing of play to be more consistent with reality; that is, we assume that the firm's choice of care is made at the time of production while the consumer's choice of care is made at the time of use. This sequential structure allows the firm to use its first-mover choice of care to influence the consumer's subsequent choice of care.

4. Obviously, to the extent that she is under-compensated even under strict liability, the consumer will exert effort on care; nevertheless, she is likely to exert too little effort as compared to the socially efficient amount. 
There are two previous papers that have modeled the sequential choice of care in a bilateral-care model of accidents, Wittman (1981) and Shavell (1983); in addition to these formal models, see Landes and Posner (1981) for a discussion of the sequential choice of care in the accident setting. Note that, in a model of accidents, in contrast with that of products liability, there is generally no market through which the consumer can transmit her preferences over safety. Wittman (1981) analyzes a model wherein two parties sequentially choose their levels of care; he argues for "marginal cost" liability rules wherein the first mover is liable for the harm plus the cost of the second-mover's socially efficient level of care (as a function of the firstmover's actual level of care). This rule (and other related rules) induces the second mover to respond in a socially efficient way to the first-mover's choice of care, and induces the first mover to choose his socially efficient level of care. Wittman does not consider the rule of strict liability with a defense of contributory negligence. Shavell (1983) analyzes an accident model in which one party (either victim or injurer) chooses her level of care first in order to influence whether or not a dangerous condition will prevail. The other party, observing the first party's choice of care and whether or not a dangerous condition prevails, chooses his level of care in order to influence the ultimate likelihood of an accident.

Our model differs from both Wittman's and Shavell's in that our parties have a buyer-seller relationship, so it is most plausible to assume that the firm chooses its level of care first (during the design or production stage) while the consumer chooses her level of care second (during use of the product). This price-mediated relationship means that, even if the consumer was expected to fail to meet her due-care standard (so that the firm expects to face no direct liability for the consumer's harm), the firm itself would invest in care in order to be able to charge a higher price for the product. Thus, we find that under strict liability with a defense of contributory negligence, the socially efficient levels of care will arise (for a given level of output), whereas for this same sequence of moves Shavell finds that strict liability with a defense of contributory negligence does not result in the socially efficient care levels.

Finally, there is some previous literature on the desirability of divided responsibilities. Shavell (1981) and Kaplow and Shavell (1994) ask whether responsibility for (1) providing incentives for care to potential injurers; and (2) effecting income redistribution can and should be divided between two 
entities, such as a legal rule specifying efficient damages and a tax authority to perform any desired redistribution. They argue that (with some qualifications) the answer is "Yes," since redistribution via either the legal system or the tax system induces distortions in the labor-leisure tradeoff, while redistribution via the legal system also induces distortions in care-taking. In our model, we take the situation of divided responsibilities for product and market performance as given, and ask whether one can identify a particular liability rule that works best in combination with agencies or other entities that are tasked with improving market performance.

\section{Sequential Choice of Care Under Proportional and Cumulative Harm}

We now detail the game between the firm and the consumer when care is bilateral and then apply it in two contexts: (1) when expected harm is proportional to the level of use of the product; and (2) when expected harm is cumulative with respect to the level of use of the product. The reason for item (1) is to illustrate the application of the game form wherein the firm moves first, followed by the consumer, in a familiar setting. In the proportional-harm case with bilateral care one would expect that strict liability for the firm (denoted as $S L$ ) will result in insufficient care-taking by the consumer, reflecting problems of moral hazard. The standard solution when the parties choose care simultaneously is to employ strict liability for the firm with a defense of contributory negligence (denoted as $S L D$ ) on the part of the consumer. This liability regime also provides the correct incentives for care under sequential choice of care, as does a regime of no liability for the firm (denoted as $N L$ ), when harm is proportional to use. We then turn to the cumulative harm case and show that, in the context of the same game form, $S L D$ is the only resilient liability policy.

\subsection{Description of the Sequential Care-Choice Game}

We envision a market that consists of a single firm and many identical consumers; the monopolist chooses quantity. ${ }^{5}$ We will assume a very simple

5. In DR2014 we discuss, at some length, the reason for using quantity as a strategic variable for the monopolist. In that paper we also consider the possibility of an $n$-firm oligopolistic industry. 
cost structure wherein the firm's cost of production and care is constant per unit of output, and the consumer's cost of care is also constant per unit of output. This means that welfare and firm profits can be expressed on a perconsumer basis and we can examine the firm's interaction with a single representative consumer.

We consider the following general protocol. Given any specific liability regime, the consumer and firm play as follows.

1. The firm simultaneously chooses a level of care, $x$, and the amount of the good to produce, $q .{ }^{6}$ The amount of the good, the level of care, and the liability regime together imply a price, $p$, for the good in the product market, to be determined as part of the analysis to come.

2. The consumer, observing $p$ and $x$, acquires an amount $q$ of the good, anticipating the amount of care she will choose to employ, $y$, in the actual use of the product.

3. The consumer chooses her level of care, $y$, possibly subject to constraints reflecting how liability is apportioned between her and the firm if, say, a defense of contributory negligence was available to the firm. This detail will be made clearer in the relevant portions of the analysis.

We will assume a full-information game, so the firm is able to anticipate correctly the consumer's marginal willingness-to-pay for the good and the care she will subsequently take in using it. Moreover, in the case of strict liability, we assume that the consumer obtains perfect compensation. We make these assumptions so that it is clear that results that distinguish the traditional (proportional-harm) model from those of the cumulative-harm model are fully attributable to the change in the modeling of expected harm, and are not due to, say, the presence of asymmetric information. We will provide a welfare analysis wherein a "central planner" chooses and dictates the levels of $q, x$, and $y$. In the analysis of the game between the firm and the consumer, we employ subgame perfection, so we first analyze the consumer's choice of $y$ for any given amount of the good purchased and any given firm investment in care-taking. We then back up a step to find the

6. The firm might instead be modeled as choosing $x$ and $q$ sequentially, but it is straightforward to show that this has no impact on the resulting choices. 
consumer's marginal willingness-to-pay for the good, and finally we find the firm's profit-maximizing choices of output and care.

In what follows we employ the following notation and functions:

$u(q)$ : The consumer's direct utility in use for $q$ units of the product; we assume that $u^{\prime}(q)>0$ and $u^{\prime \prime}(q)<0$ for all $q$.

$H(q, x, y)$ : The expected harm when the firm chooses care level $x$, the consumer (she) buys an amount $q$ of the product, and she uses care level $y$. In the proportionalharm case, $H(q, x, y) \equiv h(x, y) q$, where $h(x, y)$ is the per-unit expected harm, while for the cumulative harm case we analyze the simplest modification, $H(q, x, y) \equiv$ $h(x, y) q^{2}$, where now $h(x, y) q$ is the per-unit expected harm. Assume that $h(x, y)>0$ for all finite values of $x$ and $y$. We assume the following first and second derivative properties for $h: h_{x}<0 ; h_{y}<0 ; h_{x x}>0$; and $h_{y y}>$ 0 . That is, for each agent, their choice of care reduces expected harm but at a diminishing rate. We assume that $h_{x x} h_{y y}-\left(h_{x y}\right)^{2}>0$, so as to provide sufficient conditions for the optimization conditions to characterize maxima.

$x q, y q$ : Respectively: (1) total production cost and cost of care on the part of the firm, and (2) total effort cost for taking care on the part of the consumer.

$W(q, x, y)$ : Welfare for any given level of $(q, x, y)$. In general,

$$
W(q, x, y)=u(q)-H(q, x, y)-x q-y q .
$$

$\pi^{j}(q, x \mid y)$ : The firm's profits for any given choice of $(q, x)$ under liability regime $j(j=N L, S L$, or $S L D)$ and given the consumer's anticipated level of care, $y$. The exact form of the profit function will be specified as the analysis proceeds.

Furthermore, to avoid getting bogged down in technical complexities, we assume that all (unconstrained) optimization problems have unique interior maxima. 


\subsection{Proportional Harm with Bilateral Care}

We consider the proportional-harm model first, deriving results for the socially efficient level of output and investments by the firm and the consumer, and then we turn to the three regimes of interest, $N L, S L$, and $S L D$. We then compare these results with those found in Shavell (1983) and explain the sources of the difference in results.

3.2.1. Efficiency. Let us first find the values of $q, x$, and $y$ that characterize a socially efficient outcome (i.e., that maximize $W(q, x, y))$. Thus, we wish to examine the first-order conditions for the problem:

$$
\max _{(q, x, y)} u(q)-h(x, y) q-x q-y q
$$

these conditions are:

$$
\begin{gathered}
q: u^{\prime}(q)-h(x, y)-x-y=0, \\
x:-h_{x}(x, y) q-q=0 \\
y:-h_{y}(x, y) q-q=0 .
\end{gathered}
$$

Equation (1) states the familiar condition for efficiency of the output choice: marginal utility, $u^{\prime}(q)$, equals total marginal cost, $h(x, y)+x+y$. Equations (2) and (3) express that, with respect to either care choice alone, the marginal benefit of an increase in care should just equal the marginal cost (i.e., $-h_{z}(x, y) q=q$, for $z=x$ or $y$ at the optimum). In what follows we focus on conditions such as Equations (2) and (3) to derive the level of care for an agent such as the firm or the consumer under welfare maximization (or later under profit maximization in the context of a specific liability regime).

Consider the solutions of each individual optimality condition as an expression of the level of a decision variable as a function of the other variables. In particular, $x^{W}(q, y)$ solves Equation (2) and $y^{W}(q, x)$ solves Equation (3), where the superscript $W$ indexes the solution functions arising from the welfare maximization problem. Moreover, since $q$ is positive, we can divide by $q$ in each equation and therefore simplify the pair of functions $x^{W}(q, y)$ and $y^{W}(q, x)$ to be, respectively - and slightly abusing notation 
$-x^{W}(y)$ and $y^{W}(x)$. These functions describe the levels of care chosen by (respectively) the firm and the consumer, assuming welfare maximization, and we see that each agent's efficient level of investment in care depends upon the other agent's (arbitrary) level of care, but it does not depend upon the level of output produced (or, equivalently, quantity consumed). Given our assumptions on the function $h(x, y)$, these functions are either both upward-sloping or both downward-sloping (see the Appendix for details).

If the overall solution to Equations (1)-(3) is denoted as $\left(q^{*}, x^{*}, y^{*}\right)$, then $x^{*}=x^{W}\left(y^{*}\right)$ and $y^{*}=y^{W}\left(x^{*}\right)$, while $q^{*}$ satisfies $u^{\prime}\left(q^{*}\right)=h\left(x^{*}, y^{*}\right)+$ $x^{*}+y^{*}$. Note that it is the proportionality of expected harm (along with the linear care and production costs, $y q$ and $x q$, respectively) that provides the traditional result that the efficient investment in safety is independent of the level of output.

3.2.2. No Liability. We now consider the game between the firm and the consumer described earlier, starting with the consumer (that is, working backward). Thus, for arbitrary $x$ and $q$ we first characterize the level of care the consumer takes in using the product when the regime is no liability; as will be seen below, this level is independent of $q$ but does depend on $x$, and thus we denote it as $y^{N L}(x)$.

For fixed $x$ and $q$ the consumer's payoff reduces to the expected harm plus the cost of taking care, which should be minimized:

$$
\min _{y} h(x, y) q+y q,
$$

yielding the first-order-condition:

$$
h_{y}(x, y) q+q=0
$$

Again, for $q$ positive this means that we can solve Equation (4) to obtain the level of care to be taken by the consumer as a function of that taken by the firm, $y^{N L}(x)$. Comparing with Equation (3) we see that $y^{N L}(x)=y^{W}(x)$ for all $x$, so that the consumer's choice of care for any given level of investment in care made by the firm is the efficient amount; note that this does not automatically ensure, however, that the equilibrium level under no liability, denoted as $y^{N L *}$, is the same as $y^{*}$. 
We next turn to finding the consumer's choice of quantity to demand (which yields the consumer's marginal willingness-to-pay, or inverse demand function); this comes from the overall maximization problem for the consumer, where the consumer rationally anticipates using her best choice-of-care rule, $y^{N L}(x)$ :

$$
\max _{q} u(q)-h\left(x, y^{N L}(x)\right) q-y^{N L}(x) q-p q,
$$

where $p$ is the price paid by the consumer for each unit of the product. The first-order-condition yields the inverse demand function, $p\left(q, x \mid y^{N L}(x)\right)$ :

$$
p\left(q, x \mid y^{N L}(x)\right)=u^{\prime}(q)-h\left(x, y^{N L}(x)\right)-y^{N L}(x) .
$$

That is, the consumer deducts the (per unit) expected harm and care cost, both conditional on the continuing optimal choice of the level of care by the consumer, from the marginal utility for the good to obtain her net marginal willingness-to-pay. Thus, the firm's profit function under $N L$, denoted as $\pi^{N L}\left(q, x \mid y^{N L}(x)\right)$, is

$$
\pi^{N L}\left(q, x \mid y^{N L}(x)\right)=\left(u^{\prime}(q)-h\left(x, y^{N L}(x)\right)-y^{N L}(x)\right) q-x q .
$$

The details of the firm's choice of $x$ and $q$ are given in the Appendix. But it is clear from the profit function (and Equation (4)) that $x^{N L *}=x^{*}$ and thus $y^{N L *} \equiv y^{N L}\left(x^{N L *}\right)=y^{N L}\left(x^{*}\right)=y^{W}\left(x^{*}\right)=y^{*}$; that is, $y^{N L *}=y^{*}$. Therefore, under no liability, the firm and the consumer choose the socially efficient levels of care. Of course, since the firm is a monopoly, it will produce less than the socially efficient level of output; that is, $q^{N L *}<q^{*}$.

Proposition 1 (a) In the proportional-harm model, a regime of no liability results in the firm and the consumer using the socially efficient levels of care: $x^{N L *}=x^{*}$ and $y^{N L *}=y^{*}$.

(b) In the proportional-harm model, a regime of no liability results in the monopolist producing too little output: $q^{N L *}<q^{*}$.

\subsubsection{Strict Liability and Strict Liability with a Defense of Contributory}

Negligence. We first consider the consumer's choice of care under a regime of strict liability. For fixed $x$ and $q$ the consumer's payoff reduces to 
the expected harm plus the cost of taking care, which should be minimized; but since all harms are compensated by the firm, the consumer's objective is $\min _{y} y q$. Thus, for $q>0, y^{S L *}=0<y^{W^{*}}$. Therefore, strict liability alone cannot result in the bilateral choices of the efficient levels of care.

Now assume that the firm has a potential defense of contributory negligence on the part of the consumer, so that if the consumer took due care the firm is strictly liable, while if the consumer failed to take due care the firm is not liable for any harm (independent of the level of care taken by the firm). By due care, we mean that the consumer must take care that is at least the socially efficient level; that is, we interpret the due-care standard as requiring $y \geqslant y^{*}$. Thus, after buying an amount $q$, the consumer's care-choice problem is the following:

$$
\min _{y} y q \text { subject to } y \geqslant y^{*} \text {. }
$$

Since $q>0$, if the consumer chooses to satisfy the constraint, then $y^{S L D *}=$ $y^{*}$. Therefore, at this point in the game (where the firm's choice of $x$ is fixed and the consumer has already purchased $q$ units), the minimal expenditure on care by the consumer is $y^{*} q$.

Importantly, since the consumer chooses $y$ after the firm has chosen $x$, the consumer might find $y^{*} q$ to be a higher expected cost than if she were to violate the standard, knowing that she would then have to bear the cost of any harm. That is, should the consumer choose not to meet the duecare standard, she will face the cost of the harm plus any expenditure on care, as the regime now is equivalent to $N L$. This expected cost would be $h\left(x, y^{N L}(x)\right) q+y^{N L}(x) q$. Dividing by $q$, we can define two sets of care levels for the firm:

$$
M=\left\{x \mid y^{S L D *} \leqslant h\left(x, y^{N L}(x)\right)+y^{N L}(x)\right\},
$$

which is the set of $x$-choices by the firm such that the consumer will find it optimal to meet the due-care standard, meaning the firm is strictly liable for the harm; and

$$
m=\left\{x \mid y^{S L D *}>h\left(x, y^{N L}(x)\right)+y^{N L}(x)\right\},
$$

which is the set of $x$-choices by the firm such that the consumer will find it optimal to not meet the due-care standard, meaning the firm is not liable 
for the harm. Recall that $y^{S L D *}=y^{*}$, the socially efficient level of care; we continue to use the notation $y^{S L D *}$ to emphasize that this is the consumer's choice of care under $S L D$ when she chooses to meet the due-care standard.

Therefore, the consumer's marginal willingness-to-pay for output depends on whether $x$ is in $M$ or $m$. If $x$ is in $M$, the inverse demand function for the consumer is

$$
p\left(q, x \mid y^{S L D *}\right)=u^{\prime}(q)-y^{S L D *} .
$$

If, on the other hand, $x$ is in $m$, then the consumer adjusts her demand to reflect the fact that she will have to bear any costs associated with harm, making her inverse demand the same as would occur under $N L$ :

$$
p\left(q, x \mid y^{N L}(x)\right)=u^{\prime}(q)-h\left(x, y^{N L}(x)\right)-y^{N L}(x) .
$$

Thus, when it chooses $x$ in $M$, the firm's profit function is as if it were under $S L,{ }^{7}$ except with a consumer choice of $y=y^{S L D *}=y^{*}$ instead of $y=0$ :

$$
\begin{aligned}
\pi^{S L D}\left(q, x \mid y^{S L D *}\right) & =\left(u^{\prime}(q)-y^{S L D *}\right) q-h\left(x, y^{S L D *}\right) q-x q \text { for } x \text { in } M \\
& =\pi^{S L}\left(q, x \mid y^{*}\right) \text { for } x \text { in } M
\end{aligned}
$$

whereas it is as if it were under $N L$ when it chooses $x$ in $m$ :

$$
\begin{aligned}
\pi^{S L D}\left(q, x \mid y^{N L}(x)\right) & =\left(u^{\prime}(q)-h\left(x, y^{N L}(x)\right) q-y^{N L}(x)\right) q-x q \text { for } x \text { in } m \\
& =\pi^{N L}\left(q, x \mid y^{N L}(x)\right) \text { for } x \text { in } m .
\end{aligned}
$$

In the Appendix, we show two important results (which are fairly straightforward under proportional harm, but will be a bit more complicated under cumulative harm): (1) the socially efficient firm care level, $x^{*}$, belongs to $M ;^{8}$ and (2) the firm will always prefer to choose a level of care that will induce the consumer to meet the due-care standard. Since the firm's most-preferred care level in $M$ is $x^{*}$, and since $x^{*}$ induces the consumer to choose $y^{*}$, it follows that $S L D$ achieves the socially efficient levels of care,

7. In a manner similar to the definition of $\pi^{N L}(q, x \mid y)$, the firm's profit under $S L$ as a function of $q, x$, and $y$, is given by $\pi^{S L}(q, x \mid y)=\left(u^{\prime}(q)-y\right) q-h(x, y) q-x q$.

8. It is worth observing that, since $x^{*}$ is in the interior of $M$, small changes in $x$ near $x^{*}$ still result in $y=y^{*}$. This will then lead to $x^{*}$ being chosen by the firm, so Proposition 2 has a "built-in" robustness to small errors in the choice of $x$. 
though it also provides an inefficiently low level of output, again reflecting the firm's monopoly power.

Proposition 2 In the proportional-harm model, a regime of strict liability with a defense of contributory negligence results in the socially efficient choices of level of care $\left(x^{S L D *}=x^{*}\right.$ and $\left.y^{S L D *}=y^{*}\right)$ but a socially inefficient level of output $\left(q^{S L D *}<q^{*}\right)$.

3.2.4. Comparison with Previous Results. The primary point of comparison is with Shavell (1983). The relevant case is Shavell's discussion of the sequential-care model when the injurer moves first, wherein he finds that $S L D$ does not achieve the socially efficient levels of care. There are two substantive differences between our model and Shavell's model. First, in Shavell's model the victim's contributory negligence standard is modeled as the constraint $y \geqslant y^{W}(x)$; and second, there is no market relationship between the parties. The use of $y^{W}(x)$ as the due-care standard means that the injurer can influence the victim's due-care standard; since (in his model) a decrease in the injurer's care level causes an increase in the victim's care level, the injurer can shift some of the costs of care to the victim by choosing an inefficiently low level of care $\left(x<x^{*}\right)$. This shifting is valuable to the injurer in Shavell's model because there is no market relationship between the parties; if there were, then this cost-shifting would be reflected in a lower marginal willingness-to-pay on the part of the consumer and it would not be valuable to the firm. Our implementation of $S L D$, wherein we require $y \geqslant y^{*}$, means that for the consumer's costs of harm to be covered by the firm, she must meet a due-care standard that is the socially efficient level of care. However, should the consumer choose not to meet the due-care standard, then she will of course respond via her best-response function to the firm's choice of $x\left(y^{N L}(x)\right.$, which equals $\left.y^{W}(x)\right)$. Modeling the due-care standard in this way, we find that the firm will always choose $x$ so that the consumer meets the due-care standard by setting $y=y^{*}$, which in general is not equal to $y^{N L}(x)$ for any $x$-choices other than $x=x^{*}$. Thus, for $x \neq x^{*}$ but close enough to be in the set $M$, Shavell's victim will choose $y^{N L}(x)$ but our consumer will choose $y^{*}$. It is straightforward to show that if the accident model also used the due-care standard $y^{*}$, then $S L D$ would generate the socially efficient choice of $x=x^{*}$ as well. 


\subsection{Cumulative Harm with Bilateral Care}

We now apply the same protocol to the bilateral-care products liability problem when expected harm is cumulative and given by $H(q, x, y)=$ $h(x, y) q^{2}$. While this is a special structural form itself (which we employ for illustrative purposes), our earlier paper (DR2014) shows how, in the unilateral-care context, a more general model of harm (wherein $H(q, x)$ is simply convex in both $q$ and $x$ ) provides the result that strict liability is the only resilient liability rule (among $N L, S L$, and Negligence). We now demonstrate that strict liability with a defense of contributory negligence $(S L D)$ is a resilient liability rule (whereas $N L$ is not resilient).

3.3.1. Efficiency. When expected harm is cumulative, then the welfare function, $W(q, x, y)$ becomes

$$
W(q, x, y)=u(q)-h(x, y) q^{2}-x q-y q .
$$

Maximizing $W$ with respect to all three variables provides the following first-order conditions:

$$
\begin{gathered}
q: u^{\prime}(q)-2 h(x, y) q-x-y=0, \\
x:-h_{x}(x, y) q^{2}-q=0 \\
y:-h_{y}(x, y) q^{2}-q=0 .
\end{gathered}
$$

Unlike the conditions (Equations (2) and (3)) for the proportional-harm model, the solutions to Equations (15) and (16) are not independent of $q$. For example, dividing both sides of Equation (15) and solving for $x$ yields the socially efficient firm care level for given $(q, y)$, denoted as $x^{W}(q, y)$. Similarly, the solution to Equation (16) is denoted as $y^{W}(q, x)$. Solving Equations (15) and (16) jointly yields the functions $X^{W}(q)$ and $Y^{W}(q)$; thus, $X^{W}(q)=x^{W}\left(q, Y^{W}(q)\right)$ and $Y^{W}(q)=y^{W}\left(q, X^{W}(q)\right)$. Therefore, $X^{W}(q)$ and $Y^{W}(q)$ provide the socially efficient levels of care, but they are now conditional on the amount consumed. Among other things, this will result in modification of our model of SLD.

Solving the entire system (i.e., including Equation (14)) yields the welfare-maximizing point $\left(q^{*}, x^{*}, y^{*}\right)$, so that: (1) $x^{*}=x^{W}\left(q^{*}, y^{*}\right)$ and $y^{*}=y^{W}\left(q^{*}, x^{*}\right)$; (2) $x^{*}=X^{W}\left(q^{*}\right)$ and $y^{*}=Y^{W}\left(q^{*}\right)$; and (3) $q^{*}$ solves 
Equation (14), which can be re-expressed as

$$
u^{\prime}\left(q^{*}\right)-2 h\left(X^{W}\left(q^{*}\right), Y^{W}\left(q^{*}\right)\right) q^{*}-X^{W}\left(q^{*}\right)-Y^{W}\left(q^{*}\right)=0 .
$$

3.3.2. No Liability. Once again we start with the analysis of the $N L$ regime, and then compare it with the results from Section 3.3.1. After having purchased $q$ units, the consumer must decide on the level of care to take, which is the solution to

$$
\min _{y} h(x, y) q^{2}+y q .
$$

Note that the first-order condition is the same as that found above in Equation (16), so under $N L$ the consumer takes the same care (for any given level of care $x$ by the firm and any given $q), y^{N L}(q, x)$, as occurs in the welfare-maximization problem:

$$
y^{N L}(q, x)=y^{W}(q, x) \text { for given }(q, x) .
$$

The function $y^{N L}(q, x)$ provides the consumer's best response to the firm's choice of care, $x$, for each level of consumption, $q$. Thus, if we now use the consumer's payoff, recognizing that under $N L$ the consumer must account for the expected cost of harm, we can find the consumer's inverse demand function by solving the following problem:

$$
\max _{q} u(q)-h\left(x, y^{N L}(q, x)\right) q^{2}-y^{N L}(q, x) q-p q
$$

the only difference from Equation (5) being the quadratic quantity term, $h(x, y) q^{2}$. The first-order condition for $q$ is more complicated:

$$
\begin{aligned}
& u^{\prime}(q)-2 h\left(x, y^{N L}(q, x)\right) q-y^{N L}(q, x)-\left\{h_{y}\left(x, y^{N L}(q, x)\right) q^{2}+q\right\} \\
& \quad \times\left(\partial y^{N L}(q, x) / \partial q\right)-p=0 .
\end{aligned}
$$

However, we know that $h_{y}\left(x, y^{N L}(q, x)\right) q^{2}+q=0$ via the envelope theorem applied to the consumer's choice problem for $y$, evaluated at its solution $y^{N L}(q, x)$, in Equation (17). Therefore, Equation (20) provides us 
with the consumer's inverse demand function for the product of the firm as

$$
p\left(q, x \mid y^{N L}(q, x)\right)=u^{\prime}(q)-2 h\left(x, y^{N L}(q, x)\right) q-y^{N L}(q, x) .
$$

In comparison with Equation (6), the second term on the right is different in two ways. First, the term involving $h$ is multiplied by a " 2 " on the left and, second, it is multiplied on the right by " $q$ ". This entire term, which is the marginal expected harm, acts to rotate the inverse demand curve inward rather than to shift it downward (as occurred in Equation (6)). Alternatively put, in the proportional-harm model the marginal expected harm affects the intercept of the consumer's inverse demand function while in the cumulative-harm model, the marginal expected harm is reflected in the slope of the consumer's inverse demand function. ${ }^{9}$

Thus, the firm's profit function under no liability, denoted as $\pi^{N L}\left(q, x \mid y^{N L}(x)\right)$, is

$$
\left.\pi^{N L}\left(q, x \mid y^{N L}(q, x)\right)=\left(u^{\prime}(q)-2 h\left(x, y^{N L}(q, x)\right) q\right) q-y^{N L}(q, x)\right) q-x q .
$$

The details of the analysis are provided in the Appendix, where we also show that the firm's profit-maximizing care level (given output $q$ ), denoted by $X^{N L}(q)$, is generally not equal to the socially efficient level $X^{W}(q)$. For a given level of output $q, X^{N L}(q)$ exceeds (falls below) that which is socially efficient if and only if the cross term in the $h$-function is sufficiently small (large). ${ }^{10}$

In particular, consider the case wherein $X^{N L}(q)>X^{W}(q)$ for each $q$. This case seems intuitively reasonable, as increasing $q$ causes the firm to face (stochastically) more incidents of greater harm, so that even though it is not liable, it will want to further ameliorate the harm-effect since it is depressing the consumer's marginal willingness-to-pay (and the extent of this reduction is exacerbated by the fact that the term enters the demand

9. The exact form of the effect, $2 h(x, y) q$, reflects the quadratic form assumption for $H(q, x, y)$. More generally, this marginal effect is $H_{q}(q, x, y)$, and one would normally expect that $H_{q}(q, x, y)$ is increasing in $q$, again affecting the slope of the inverse demand function; see the Technical Appendix for DR2014 for details in the unilateralcare case.

10. More specifically, $X^{N L}(q)(>,=,<) X^{W}(q)$ if and only if $\mathrm{d} X^{W}(q) /$ $\mathrm{d} q(>,=,<) 0$ if and only if $h_{y y}\left(h_{x} / h_{y}\right)(>,=,<) h_{x y}$. 
curve both at a rate of twice the average expected harm, and proportional to the quantity consumed).

This over-supply (or under-supply) of care by the firm, holding $q$ fixed, is equivalent to Spence's (1975) analysis of the over- or under-provision of quality by a monopolist, which can be thought of as a model with no liability (since the issue there is product quality, not safety). This equivalence occurs because, in our model, the continuation game for the consumer under $N L$ is represented by the best response function $y^{N L}(q, x)$, thereby making the inverse demand function (as shown in Equation (21) above) a function of $q$ and $x$ (but not of $y$ ). That is, under constant marginal costs of production (as used in Spence and as employed here), the question of over- or undersupply of quality (care) is fully determined by the sign of the cross derivative $P_{q x}(q, x)$ where, here, $P(q, x) \equiv p\left(q, x \mid y^{N L}(q, x)\right) .{ }^{11}$

3.3.3. Strict Liability with a Defense of Contributory Negligence. We proceed by skipping any discussion of $S L$ alone (due to the moral hazard problem on the consumer side, as noted earlier, $y^{S L *}=0$ ) and analyze the $S L D$ case instead. We capture the notion of the consumer meeting the duecare standard by requiring that:

$$
y \geqslant Y^{W}(Q)
$$

where $Q$ is the common level of consumption of each other ("representative") consumer in the market, and therefore not under the control of the particular consumer at hand. Inequality (23) means that: (1) when choosing the quantity to demand, the consumer does not influence the amount $Q$ and (2) when the monopoly firm is choosing the amount of output to produce per consumer, then $Q=q$, so that the firm's choice of output does affect the due-care standard that the consumer must subsequently meet to avoid being contributorily negligent. In the proportional-harm model, $Y^{W}(Q)=y^{*}$ for all possible levels of $Q$, but in the cumulative-harm model, since output and care are inextricably linked, this is not possible. Nevertheless, we will see that using $Y^{W}(Q)$ as the due-care standard for the consumer will result in

11. Using Equation (6) it is immediate that in the proportional harm case, the cross-derivative is $P_{q x}=0$, meaning that under proportional harm there is neither an over- nor under-supply of $x$. 
both the firm and the consumer choosing the socially efficient care levels for a given level of output. But this is exactly the target behavior under a resilient liability rule; the efficient adjustment of output is viewed as the responsibility of other agencies and/or courts tasked with enhancing market performance.

Thus, the cumulative-harm version of Equation (8) is

$$
\min _{y} y q \text { subject to } y \geqslant Y^{W}(Q) \text {. }
$$

If the consumer meets the due-care standard, her care cost is $Y^{W}(Q) q$ and therefore her payoff at the point of choosing how much of the product to buy at a price $p$ is:

$$
u(q)-Y^{W}(Q) q-p q,
$$

since by meeting the due-care standard, the consumer is in a regime of strict liability on the part of the firm for all harm. Maximizing the function in (25) yields the (inverse) demand function:

$$
p\left(q, x \mid Y^{W}(Q)\right)=u^{\prime}(q)-Y^{W}(Q) .
$$

On the other hand, if the consumer does not meet the due-care standard, her payoff is as in the $N L$ case:

$$
u(q)-h\left(x, y^{N L}(q, x)\right) q^{2}-y^{N L}(q, x) q-p q,
$$

and the (inverse) demand function is the same as under $N L$, shown in Equation (21) above:

$$
p\left(q, x \mid y^{N L}(q, x)\right)=u^{\prime}(q)-2 h\left(x, y^{N L}(q, x)\right) q-y^{N L}(q, x) .
$$

As in the proportional-harm analysis, it is the firm's choice of $x$ (for any given $q$ and $Q$ ) that determines whether it is optimal for the consumer to meet, or not meet, the due-care standard. The consumer decides whether to meet that standard by comparing the cost of meeting the standard and being fully compensated (that is, $Y^{W}(Q) q$ ) with the cost of choosing a (lower) best-response level of care and bearing the expected harm (that is, $\left.h\left(x, y^{N L}(q, x)\right) q^{2}+y^{N L}(q, x) q\right)$. 
Hence, we again define two sets for the consumer, now indexed by $Q$ and $q$ :

$$
M^{C}(Q, q)=\left\{x \mid Y^{W}(Q) q \leqslant h\left(x, y^{N L}(q, x)\right) q^{2}+y^{N L}(q, x) q\right\}
$$

and

$$
m^{C}(Q, q)=\left\{x \mid Y^{W}(Q) q>h\left(x, y^{N L}(q, x)\right) q^{2}+y^{N L}(q, x) q\right\} .
$$

For example, if $x$ is in $M^{C}(Q, q)$, then the consumer chooses to meet the standard, $y=Y^{W}(Q)$, and the firm is subject to strict liability. Since we are analyzing a monopolist facing identical consumers, then $Q=q$ from the firm's perspective (the amount consumed will be the same for all consumers), so the firm is choosing $x$ in $M^{C}(Q, q)$ with $Q=q$. For convenience, let $M(q) \equiv M^{C}(q, q)$ be the set of $x$-values that induce the consumer to meet the standard for a given $q$. Similarly, let $m(q) \equiv m^{C}(q, q)$ be the set of $x$-values that induce the consumer to not meet the standard for a given $q$.

In the Appendix we show the following: ${ }^{12}$

$$
\max _{x \in M(q)} \pi^{S L}\left(q, x \mid Y^{W}(q)\right)>\max _{x \in m(q)} \pi^{N L}\left(q, x \mid y^{N L}(q, x)\right),
$$

where the left-hand side of the inequality in (29) is the profit for the firm if it chooses $x$ in $M(q)$, thereby inducing the consumer to meet the standard, and the right-hand side is the profit for the firm if it instead chooses $x$ in $m(q)$, thereby inducing the consumer to not meet the standard. Inequality (29) states that, for every choice of $q$ by the firm, it is more profitable to choose $x$ in $M(q)$ than in $m(q)$. Moreover, we also show in the Appendix that the firm's choice of $x$, denoted as $X^{\operatorname{SLD}}(q)$, is equal to $X^{W}(q)$, so that the result of using a regime of strict liability with a defense of contributory negligence is that the levels of care chosen are socially efficient, for any given value of $q$ : $\left(X^{S L D}(q), Y^{S L D}(q)\right)=$ $\left(X^{W}(q), Y^{W}(q)\right)$.

12. In a manner similar to the definition of $\pi^{N L}(q, x \mid y)$, the firm's profit as a function of $q, x$, and $y$, under $S L$ is given by $\pi^{S L}(q, x \mid y)=\left(u^{\prime}(q)-y\right) q-h(x, y) q^{2}-x q$. 


\subsubsection{Summary of Results for the Cumulative Harm Model and the} Resilient Liability Regime. We summarize the results for the $N L$ and SLD models in the following proposition:

Proposition 3 When expected harm is cumulative:

(a) Under $N L$, the firm generally does not choose the socially efficient level of care for any given level of output, $q$. Although the consumer's best response function is $y^{N L}(q, x)=y^{W}(q, x)$ for given $(q, x)$, since the firm's choice $X^{N L}(q)$ is not socially efficient, neither is the consumer's choice $Y^{N L}(q)=y^{N L}\left(q, X^{N L}(q)\right) \neq Y^{W}(q)$.

(b) Under $S L D$ the firm will always find it optimal to choose a level of care that induces the consumer to choose the socially efficient level of care for any given $q: Y^{S L D}(q)=Y^{W}(q)$. Moreover, under $S L D$ the firm will, for any given level of $q$, choose the socially efficient level of care: $X^{S L D}(q)=X^{W}(q)$.

Again, possession of monopoly power means that $S L D$ does not result in the socially efficient level of output; rather, output under $S L D$ is inefficiently low. However, we observe that $S L D$ is a resilient policy in that (1) it is robust to the unobservability of the firm's level of care, although it clearly requires that whether or not the consumer took due care must be observable and (2) as another agency tasked with improving market performance undertakes policies (such as output subsidies) that increase $q$ towards its socially efficient level, $q^{*}$, then the equilibrium care levels, $\left(X^{S L D}(q), Y^{S L D}(q)\right)=\left(X^{W}(q), Y^{W}(q)\right)$, also move toward their socially efficient levels $x^{*}=X^{W}\left(q^{*}\right)$ and $y^{*}=Y^{W}\left(q^{*}\right)$. The liability regime $N L$ does not display this robustness to the unobservability of the firm's level of care, nor does it coordinate well with another agency or body of law that is focused on improving market performance, since (in general) profitmaximizing care under no liability, $X^{N L}(q)$, does not equal socially efficient care, $X^{W}(q)$, when harm is cumulative.

\section{Conclusions}

In a previous paper (DR2014), we re-examined the unilateral care model for products liability, allowing for cumulative expected harm (that is, when expected harm is increasing at a greater-than-proportional rate); we found 
that the traditional results wherein the level of care was independent of both the liability regime and the level of output (and therefore, of market structure) were changed in a substantial manner: with cumulative harm, choice of care and choice of output level by the firm were inextricably interrelated. This fact, and the recognition that issues of market performance (e.g., enhancement of competition) and of product performance (particularly, liability) are generally the province of separate areas of law and/or separate agencies, led us to setting criteria for selecting a liability regime. We called the collection of criteria (that the regime should be robust to movements off of the domain of the analysis and that it should be consistent with market performance efforts aimed at moving the market and product in the direction of social efficiency) "resilience" and showed that strict liability was the unique resilient policy for the unilateral care model.

However, when we extend consideration to the bilateral care case, strict liability by itself leads to the possibility of moral hazard on the part of the product's user. Since the firm embeds the investment in care before the sale, and the consumer makes a choice about the amount of care in use to employ after the sale, under strict liability the consumer is tempted to take zero care since she will be fully compensated by the firm anyway: strict liability, alone, is no longer resilient. Significantly, a standard modification is resilient: in comparison with no liability, strict liability with a defense of contributory negligence is the unique resilient policy for the liability regime. ${ }^{13}$

The analysis in the paper employs a three-stage model in an attempt to model realistically the sequence of choices made by product producers and product users. In the first stage, the firm picks the amount of output to produce and the level of care to provide. In the second stage, the consumer chooses an amount of the product to buy, while in the third stage she chooses a level of care to take in the use of the product. Working from the end backwards we derive a subgame perfect equilibrium wherein under $S L D$ we find that the firm would choose a level of care that would induce the consumer

13. A regime of simple negligence, and a regime of negligence with a defense of contributory negligence, fail to induce socially efficient care on the part of the firm and the consumer, for a given level of output; see the Appendix for a discussion. Thus, they fail to be resilient regimes even if firm care is observable by the consumer prior to purchase and by the court after purchase. 
to choose her efficient level of care; the firm's choice would also be the efficient level of care for the firm given the level of output on the market. Thus, if those agents/agencies in the economy focused on market performance work to enhance market performance (e.g., provide a subsidy that induces output to rise), then product performance will also improve and move toward the socially efficient level.

\section{Extensions}

In DR2014, we also considered alternative market structures (here we have examined only monopoly), finding how the intuitions from the onefirm model extend as we increase the number of firms and allow for alternative manners in which the potential for harm arising from the multiple possible sources may be aggregated. This is a potential extension for the bilateral care case which we have not pursued herein, due to the added space such work would require for exposition. Other possible avenues for extension would include: (1) harms to third parties (i.e., not those purchasing the product for own use) and (2) non-market torts involving bilateral care.

\section{Appendix}

This appendix contains details of the analysis; in addition, it describes some comparative statics results that may be of interest to some readers, but that are not necessary to make the main points regarding efficiency.

\section{An Example of an h-function}

We note a convenient example of our function $h(x, y)$. Let $h(x, y)=$ $A /\left(x^{r}+y^{r}\right)^{1 / r}$ with $r<1, A$ an appropriate constant, and at least one of $x$ and $y$ are positive; the denominator of $h$ is the constant elasticityof-substitution (CES) model of utility or production used in microeconomic analysis. ${ }^{14}$ Common special cases of $h$ are $A /(x y)^{1 / 2}$ when $r=0$;

14. In the limiting case wherein $r=1,\left(h_{x y}\right)^{2}=h_{x x} h_{y y}$, so strict convexity is lost. This means that $x$ and $y$ are perfect substitutes in the "production function" for expected harm, so there will not be a unique solution for the pair. Hence, we restrict $r$ to be less than 1 . 
$A(x+y) / x y$ when $r=-1$; and $A / \min \{x, y\}$ when $r \rightarrow-\infty$. With some work one can show that $h_{x y}(>,=,<) 0$ as $r(>,=,<)-1$.

Comparative Statics of Socially Efficient Care in the Case of Proportional Harm

If we differentiate Equation (2) and collect terms, then we find that

$$
\mathrm{d} x^{W}(y) / \mathrm{d} y=-h_{x y} / h_{x x} .
$$

Similarly, if we differentiate Equation (3) and collect terms, then we find that

$$
\mathrm{d} y^{W}(x) / \mathrm{d} x=-h_{x y} / h_{y y} .
$$

Since we have assumed that $h_{x x}>0$ and $h_{y y}>0$, then the sign of the crossterm $h_{x y}$ (which indicates how $x$ and $y$ substitute so as to provide any constant level of $h(x, y))$ determines the sign of the slope of $x^{W}(y)$ as well as the sign of the slope of $y^{W}(x)$.

The signs of these derivatives matter because they tell us whether the solution to the overall system, $\left(q^{*}, x^{*}, y^{*}\right)$ involves $\left(x^{*}, y^{*}\right)$ occurring where $x^{W}(y)$ and $y^{W}(x)$ are downward-sloping or upward-sloping; as we will see, either case can occur, but importantly, both curves have the same direction of slope. As an example to illustrate how the properties of $h(x, y)$ influence the slopes of $x^{W}(y)$ and $y^{W}(x)$, recall the example introduced earlier: $h(x, y)=A /\left(x^{r}+y^{r}\right)^{1 / r}$ with $r<1$. From Equations (A1) and (A2), we see that the functions $x^{W}(y)$ and $y^{W}(x)$ are downward- (upward-) sloping as $r(>,<)-1$.

Analysis of Profit-Maximization under No Liability in the Case of Proportional Harm

The first-order conditions for the problem $\max _{q, x} \pi^{N L}\left(q, x \mid y^{N L}(x)\right)$ are as follows:

$$
\begin{aligned}
q & : u^{\prime}(q)+u^{\prime \prime}(q) q-h\left(x, y^{N L}(x)\right)-y^{N L}(x)-x=0, \\
x & :-h_{x}\left(x, y^{N L}(x)\right) q-h_{y}\left(x, y^{N L}(x)\right) q\left(\mathrm{~d} y^{N L}(x) / \mathrm{d} x\right) \\
& -q\left(\mathrm{~d} y^{N L}(x) / \mathrm{d} x\right)-q=0 .
\end{aligned}
$$


Equation (A4) can be simplified substantially by recognizing that, from the derivation of $y^{N L}(x)$ from Equation (4), we know the following:

$$
\left[h_{y}\left(x, y^{N L}(x)\right) q+q\right]\left(\mathrm{d} y^{N L}(x) / \mathrm{d} x\right)=0,
$$

so that Equation (A4) can be more simply written as

$$
x:-h_{x}\left(x, y^{N L}(x)\right) q-q=0 .
$$

That is, the conditions characterizing the firm's optimal output and care decisions are Equations (A3) and (A4'). Since (from the earlier analysis) $y^{N L}(x)=y^{W}(x)$, this means that when we compare Equation (A4') and Equation (2) from the welfare discussion earlier, we must have that Equation $\left(\mathrm{A}^{\prime}\right)$ is solved at $x^{N L *}=x^{*}$. Furthermore, $y^{N L *} \equiv y^{N L}\left(x^{N L *}\right)=$ $y^{N L}\left(x^{*}\right)=y^{W}\left(x^{*}\right)=y^{*}$; that is, $y^{N L *}=y^{*}$. Therefore, under no liability, the firm and the consumer choose the socially efficient levels of care.

Of course, a glance at Equations (1) and (A3) indicates that $q^{N L *} \neq q^{*}$, since there is an extra term in Equation (1). To see what is happening, substitute $\left(q^{*}, x^{N L *}, y^{N L *}\right)$ into the left-hand side of Equation (A3), which yields (after re-arrangement):

$$
u^{\prime}\left(q^{*}\right)-h\left(x^{N L *}, y^{N L *}\right) q^{*}-y^{N L *}-x^{N L *}+u^{\prime \prime}\left(q^{*}\right) q^{*} .
$$

The first four terms in expression (A5) add to zero from Equation (1) and the result that $\left(x^{N L *}, y^{N L *}\right)=\left(x^{*}, y^{*}\right)$, but the fifth term is negative by the assumptions that $u^{\prime \prime}(q)<0$ for all $q$ and that $q^{*}>0$. That is, the marginal profit from one more unit of output under $N L$, evaluated at the socially efficient outcome $\left(q^{*}, x^{*}, y^{*}\right)$, is negative, so the firm would want to reduce output from $q=q^{*}$. Thus, the solution to Equation (A3) necessarily implies that $q^{N L *}<q^{*}$; this is a reflection of the monopoly power of the firm.

Finally, since $x^{N L}(y)=x^{W}(y)$ and $y^{N L}(x)=y^{W}(x)$, then the slope properties of the two $N L$ care functions are the same as under welfare maximization: the two functions are downward- (upward-) sloping as $h_{x y}$ is greater than (respectively, less than) zero. 
Analysis of Proft-Maximization under Strict Liability with a Defense of Contributory Negligence in the Case of Proportional Harm

Claim $1 x^{*} \in M$. That is, efficient care on the part of the firm will induce efficient care on the part of the consumer.

Proof. Since $y^{*}=y^{N L}\left(x^{*}\right)$, and $h\left(x^{*}, y^{*}\right)>0$ then $h\left(x^{*}, y^{N L}\left(x^{*}\right)\right)+$ $y^{N L}\left(x^{*}\right)=h\left(x^{*}, y^{*}\right)+y^{*}>y^{*}$. Thus, $x^{*} \in M$.

Claim 2 For all $q$, the firm will always prefer to choose a level of care that will induce the consumer to meet the due-care constraint. That is, for each $q$ :

$$
\max _{x \in M} \pi^{S L}(q, x \mid y *)>\max _{x \in m} \pi^{N L}\left(q, x \mid y^{N L}(x)\right) .
$$

Proof. $\max _{x, y} \pi^{S L}(q, x \mid y)$ provides the maximum profits for the firm under $S L$ if the firm can choose both $x$ and $y$ without restriction. It is straightforward to see that it would choose $x=x^{*}$ and $y=y^{*}$. Thus, for each $q$, the firm can achieve this unrestricted maximum by choosing $x^{*} \in$ $M$, so

$$
\max _{x \in M} \pi^{S L}\left(q, x \mid y^{*}\right)=\max _{x, y} \pi^{S L}(q, x \mid y) .
$$

From the results of the $N L$ analysis earlier, it is straightforward to see that:

$$
\max _{x, y} \pi^{S L}(q, x \mid y)=\max _{x} \pi^{N L}\left(q, x \mid y^{N L}(x)\right) .
$$

Note that the right-hand-side of Equation (A8) is also optimized at $\left(x^{*}, y^{*}\right)$. However, the right-hand side of Equation (A8) strictly exceeds the righthand side of Equation (A6), since $x^{*}$ is not in $m$, and requiring $x \in m$ must therefore be a restriction on the optimal value to the problem.

Comparative Statics of Socially Efficient Care in the Case of Cumulative Harm

One can readily show that:

$$
\partial x^{W}(q, y) / \partial y=-h_{x y} / h_{x x} \quad \text { and } \quad \partial y^{W}(q, x) / \partial x=-h_{x y} / h_{y y},
$$


meaning that the signs of the slopes (holding $q$ constant) of the $x^{W}$ and $y^{W}$ functions are again determined by the sign of $\left(-h_{x y}\right)$. One can also show that:

$$
\mathrm{d} X^{W}(q) / \mathrm{d} q>0 \text { or } \mathrm{d} Y^{W}(q) / \mathrm{d} q>0 \text { or both }>0 \text { but not both }<0 .
$$

More precisely, the condition on the slope is determined by the magnitude and sign of $h_{x y}$ :

$$
\begin{array}{lll}
\mathrm{d} X^{W}(q) / \mathrm{d} q>0 & \text { if and only if } & h_{x y}<h_{y y}\left(h_{x} / h_{y}\right) \\
\mathrm{d} Y^{W}(q) / \mathrm{d} q>0 & \text { if and only if } & h_{x y}<h_{x x}\left(h_{y} / h_{x}\right) .
\end{array}
$$

Thus, for example, if $h_{x y}<0$, then both $X^{W}(q)$ and $Y^{W}(q)$ are increasing in $q$ since the above conditions are always met. When $h_{x y}>0$, then jointly requiring the above conditions is equivalent to the requirement that: $h_{x y}<\min \left\{h_{x x}\left(h_{y} / h_{x}\right), h_{y y}\left(h_{x} / h_{y}\right)\right\}$. This restriction on the magnitude of $h_{x y}$ is stronger than that associated with the convexity of $h$ (i.e., $\left.h_{x x} h_{y y}-\left(h_{x y}\right)^{2}>0\right)$.

Returning to our earlier example wherein $h(x, y)=A /\left(x^{r}+y^{r}\right)^{1 / r}$ with $r<1$, one can show that the above condition is always met: for this harm function, $X^{W}(q)$ and $Y^{W}(q)$ are always increasing in $q$ for all values of $r<1$. Thus, even though $x$ and $y$ may for some parameter values (i.e., values of $r$ ) behave like substitutes (that is, one variable increases while the other decreases) and for other parameter values behave like complements (they both increase or they both decrease), their socially efficient values always increase if the amount consumed rises. If we think of consumption (q) increasing as a result of, say, output subsidies to the firm, then the planner would also choose to increase both the firm's and the consumer's prescribed levels of care.

Analysis of Profit-Maximization under No Liability in the Case of Cumulative Harm

The first-order conditions for the problem $\max _{q, x} \pi^{N L}\left(q, x \mid y^{N L}(q, x)\right)$ are as follows (incorporating the first-order condition for the consumer's 
optimal choice of $y$ ):

$$
\begin{aligned}
q & : u^{\prime}(q)+u^{\prime \prime}(q) q-4 h\left(x, y^{N L}(q, x)\right) q-y^{N L}(q, x)-h_{y}\left(x, y^{N L}(x)\right) \\
& \times q^{2}\left(\partial y^{N L}(q, x) / \partial q\right)-x=0, \\
x & :-2 h_{x}\left(x, y^{N L}(q, x)\right) q^{2}-h_{y}\left(x, y^{N L}(q, x)\right) q^{2}\left(\partial y^{N L}(q, x) / \partial x\right)-q=0 .
\end{aligned}
$$

Let the solution to Equation (A12) be denoted as $X^{N L}(q)$; note that only $q$ appears because $y$ has been evaluated at $y^{N L}(q, x)$.

We first want to know how $X^{N L}(q)$ is related to $X^{W}(q)$. To see, re-write Equation (A12) as follows:

$$
\begin{aligned}
-h_{x}\left(x, y^{N L}(q, x)\right) q^{2}-q= & h_{x}\left(x, y^{N L}(q, x)\right) q^{2}+h_{y}\left(x, y^{N L}(q, x)\right) \\
& \times q^{2}\left(\partial y^{N L}(q, x) / \partial x\right) .
\end{aligned}
$$

The left-hand side of Equation (A13) is the same form as the left-hand side of Equation (15). If we evaluate the left-hand side of Equation (A13) at $x=$ $X^{W}(q)$, then the left-hand side would be zero (as seen from Equation (15)) if $X^{N L}(q)=X^{W}(q)$. However, in general, the right-hand side of Equation (A13) is not zero, so this means that in general, $X^{N L}(q) \neq X^{W}(q)$.

Since $h_{x}<0$ and $h_{y}<0$, then if $\partial y^{N L}(q, x) / \partial x>0$ (i.e., if $h_{x y}<0$ ), then the right-hand side of Equation (A13) is negative, meaning that it must be that $X^{N L}(q)>X^{W}(q)$. Note that even if $h_{x y}>0$, if $h_{x y}<h_{y y}\left(h_{x} / h_{y}\right)$ then $X^{N L}(q)>X^{W}(q)$. From the earlier discussion of efficiency,

$$
h_{x y}<h_{y y}\left(h_{x} / h_{y}\right) \text { if and only if } \mathrm{d} X^{W}(q) / \mathrm{d} q>0 .
$$

Alternatively, $X^{N L}(q)<X^{W}(q)$ if and only if $\mathrm{d} X^{W}(q) / \mathrm{d} q<0$.

Analysis of Proft-Maximization under Strict Liability with a Defense of Contributory Negligence in the Case of Cumulative Harm

Claim $3 X^{W}(q) \in M(q)$ for all $q$. That is (for given $q$ ), efficient care on the part of the firm will induce efficient care on the part of the consumer.

Proof. Recall that $M(q)=\left\{x \mid Y^{W}(q) q \leq h\left(x, y^{N L}(q, x)\right) q^{2}+\right.$ $\left.y^{N L}(q, x) q\right\}$. Since $y^{N L}(q, x)=y^{W}(q, x)$, then $y^{N L}\left(q, X^{W}(q)\right)=$ 
$y^{W}\left(q, X^{W}(q)\right)=Y^{W}(q)$. Thus, the inequality above becomes: $Y^{W}(q) q \leq h\left(X^{W}(q), Y^{W}(q)\right) q^{2}+Y^{W}(q) q$, which clearly holds for all $q$ since $h(x, y)>0$ for all $(x, y)$. Thus, $X^{W}(q) \in M(q)$ for all $q$.

Claim 4 For all $q$, the firm always prefers to choose a level of care that will induce the consumer to meet the due-care standard. That is, for each $q$ :

$$
\max _{x \in M(q)} \pi^{S L}\left(q, x \mid Y^{W}(q)\right)>\max _{x \in m(q)} \pi^{N L}\left(q, x \mid y^{N L}(q, x)\right) .
$$

Proof. If the firm chooses $q$, and $x$ in $M(q)$, so as to maximize its profit, then it would operate in a regime of $S L$ :

$$
\begin{aligned}
& \max _{q, x \in M(q)} \pi^{S L}\left(q, x \mid Y^{W}(q)\right) \\
& \quad=\max _{q, x \in M(q)}\left(u^{\prime}(q)-Y^{W}(q)\right) q-h\left(x, Y^{W}(q)\right) q^{2}-x q .
\end{aligned}
$$

If the consumer does not meet the standard then the consumer picks $y=$ $y^{N L}(q, x)$, and the firm does not face liability for any harm, though the expected cost of the harm affects the demand for the product. Therefore, if the firm chooses $q$, and $x$ in $m(q)$, so as to maximize its profit, then it would operate in a regime of $N L$ :

$$
\begin{aligned}
& \max _{q, x \in m(q)} \pi^{N L}\left(q, x \mid y^{N L}(q, x)\right) \\
& \quad=\max _{q, x \in m(q)}\left(u^{\prime}(q)-2 h\left(x, y^{N L}(q, x)\right) q-y^{N L}(q, x)\right) q-x q .
\end{aligned}
$$

Now, consider the inner optimization problems (that is, for arbitrary $q$ ):

$$
\max _{x \in M(q)} \pi^{S L}\left(q, x \mid Y^{W}(q)\right) \text { and } \max _{x \in m(q)} \pi^{N L}\left(q, x \mid y^{N L}(q, x)\right) .
$$

The first optimization problem's first-order condition for arbitrary (unrestricted) $x$ is

$$
-h_{x}(x, y) q^{2}-q=0 \text { with } y=Y^{W}(q) .
$$

Denote the solution to the first part of the condition in (A16) as $x^{S L D}(q, y)$. This is the same first-order condition as Equation (15) in the main text, so $x^{S L D}(q, y)=x^{W}(q, y)$. In particular, the solution to the $x$-unrestricted maximization problem is evaluated at $y=Y^{W}(q)$, so $x^{S L D}\left(q, Y^{W}(q)\right)=$ $x^{W}\left(q, Y^{W}(q)\right)$, but this latter term is $X^{W}(q)$, which belongs to $M(q)$. 
Therefore, the solution to the restricted problem (wherein $x$ is restricted to belong to $M(q))$ is also

$$
X^{S L D}(q) \equiv x^{S L D}\left(q, Y^{W}(q)\right)=X^{W}(q)
$$

In other words, the optimal level of care by the firm under $S L D$, when care is restricted to induce consumers to choose to meet the due-care standard, is the socially efficient level of care, $X^{W}(q)$, for any given $q$.

Next, we claim that the firm prefers to choose from $M(q)$ rather than from $m(q)$ : the firm prefers to induce the consumer to meet the due care constraint. To see this, consider the optimization problem:

$$
\max _{x, y} \pi^{S L}(q, x \mid y)=\max _{x, y} u^{\prime}(q) q-h(x, y) q^{2}-x q-y q .
$$

In this optimization problem $q$ is given but the firm is free to pick both $x$ and $y$. It is straightforward to show that the optimal solution is $\left(X^{W}(q), Y^{W}(q)\right)$, so that:

$$
\max _{x \in M(q)} \pi^{S L}\left(q, x \mid Y^{W}(q)\right)=\max _{x, y} \pi^{S L}(q, x \mid y) .
$$

Moreover,

$$
\begin{aligned}
& \max _{x, y} \pi^{S L}(q, x \mid y)>\max _{x, y} \pi^{N L}(q, x \mid y) \\
& \quad=\max _{x, y}\left(u^{\prime}(q)-2 h(x, y) q\right) q-x q-y q,
\end{aligned}
$$

where the inequality follows because of the 2 multiplying the $h(x, y)$ term in $\pi^{N L}(q, x \mid y)$ versus a 1 multiplying the same term in $\pi^{S L}(q, x \mid y)$. Finally, since requiring $x$ to be in $m(q)$ and allowing the consumer to best-respond via $y^{N L}(q, x)$ are both restrictions, it must be that:

$$
\max _{x, y} \pi^{N L}(q, x \mid y) \geqslant \max _{x \in m(q)} \pi^{N L}\left(q, x \mid y^{N L}(q, x)\right) .
$$

Therefore, we have shown that the firm's profits are higher when it faces strict liability for harm by inducing the consumer to meet the due-care standard by choosing an $x$ in $M(q)$, than when it faces no liability for harm by inducing contributory negligence on the part of the consumer by choosing an $x$ in $m(q)$. 
Simple Negligence, and Negligence with a Defense of Contributory Negligence, Do Not Induce $\left(X^{W}(q), Y^{W}(q)\right)$

First consider a simple negligence rule wherein the firm's due care standard is $X^{W}(q)$. We argue that it cannot be an equilibrium for the firm to just meet this standard (and thereby induce socially efficient care on the part of the consumer as well). Given that the firm at least meets its due care standard (by choosing $x \geqslant X^{W}(q)$ ), then the consumer will bear the full expected harm and things will play out as in $N L$ above. In particular, it was shown above that $X^{N L}(q)>X^{W}(q)$ as long as $\mathrm{d} X^{W}(q) / \mathrm{d} q>0$ (sufficient conditions on $h(x, y)$ for this to hold are given above, and it seems like the most plausible case: as the firm sells more output, it is socially efficient to increase the firm's care). Thus, when faced with the negligence standard $X^{W}(q)$, if the firm complies, then it will actually over-invest in care; and if it fails to comply, then it will under-invest in care.

Now consider a regime of negligence with a defense of contributory negligence, with due care standards of $X^{W}(q)$ and $Y^{W}(q)$ for the firm and consumer, respectively. Suppose that the firm just meets its due care standard, which in turn induces the consumer to just meet her due care standard (since $\left.y^{N L}(q, x)=y^{W}(q, x)\right)$. In this case, the socially efficient care levels are being chosen but, again, the consumer is discounting her marginal willingness-to-pay for the product at the marginal expected harm $2 h(x, y) q$ because she faces the full expected harm. If the firm were to reduce its choice of care to $X^{W}(q)-\varepsilon$ (and the consumer observes this choice prior to purchase) the firm would become liable provided the consumer maintained her care at $Y^{W}(q)$. The consumer would prefer to maintain $y=Y^{W}(q)$ and avoid liability; moreover, anticipating compensation from the firm, she would not discount her marginal willingness-to-pay for the product. Thus the firm would face marginal liability-related costs based on the average expected harm $h(x, y) q$. As in the unilateral-care case, the firm prefers a regime of strict liability to a negligence regime (both augmented with a defense of contributory negligence).

\section{References}

Daughety, Andrew F., and Jennifer F. Reinganum. 2006. "Markets, Torts and Social Inefficiency," 37 RAND Journal of Economics 300-23. 
Daughety, Andrew F., and Jennifer F. Reinganum. 2014. "Cumulative Harm and Resilient Liability Rules for Product Markets," Journal of Law, Economics, and Organization: available online at http://jleo.oxfordjournals.org/ content/early/2012/12/08/jleo.ews045.full.pdf + html.

Kaplow, Louis, and Steven Shavell. 1994. "Why the Legal System is Less Efficient than the Income Tax in Redistributing Income," 23 Journal of Legal Studies $667-81$.

Landes, William M., and Richard A. Posner. 1981. "The Positive Economic Theory of Tort Law," 15 Georgia Law Review 851-924.

Landes, William M., and Richard A. Posner. 1985. "A Positive Economic Analysis of Products Liability,” 14 Journal of Legal Studies 535-67.

Marino, Anthony M. 1988. "Monopoly, Liability and Regulation," 54 Southern Economic Journal 913-27.

Polinsky, A. Mitchell. 1980. "Strict Liability vs. Negligence in a Market Setting," 70 American Economic Review 363-67.

Polinsky, A. Mitchell, and William P. Rogerson. 1983. "Products Liability, Consumer Misperceptions, and Market Power," 14 RAND Journal of Economics 581-89.

Shavell, Steven. 1980. "Strict Liability versus Negligence," 9 Journal of Legal Studies $1-25$.

Shavell, Steven. 1981. "A Note on Efficiency vs. Distributional Equity in Legal Rulemaking: Should Distributional Equity Matter Given Optimal Income Taxation?," 71 American Economic Review 414-18.

Shavell, Steven. 1983. "Torts in Which Victim and Injurer Act Sequentially," 26 Journal of Law and Economics 589-612.

Shavell, Steven. 2007. Liability for Accidents. Chapter 2 in Handbook of Law and Economics, Vol. 1, ed. A. Mitchell Polinsky and Steven Shavell. Amsterdam: Elsevier.

Sheshinski, Eytan. 1976. "Price, Quality and Quantity Regulation in Monopoly Situations," 43 Economica 127-37.

Spence, A. Michael. 1975. "Monopoly, Quality, and Regulation," 6 Bell Journal of Economics 417-29.

Spulber, Daniel. 1989. Regulation and Markets. Cambridge, MA: The MIT Press.

Wittman, Donald. 1981. "Optimal Pricing of Sequential Inputs: Last Clear Chance, Mitigation of Damages, and Related Doctrines in the Law," 10 Journal of Legal Studies 65-91. 